\title{
Aharonov-Bohm phase for an electromagnetic wave background
}

\author{
Max Bright $^{1, \mathrm{a}}$, Douglas Singleton ${ }^{1,2, \mathrm{~b}}$, Atsushi Yoshida ${ }^{3,4, \mathrm{c}}$ \\ ${ }_{1}^{1}$ Department of Physics, California State University Fresno, Fresno, CA 93740-8031, USA \\ 2 ICTP South American Institute for Fundamental Research, UNESP-Univ. Estadual Paulista, São Paulo, SP 01140-070, Brazil \\ ${ }^{3}$ Department of Physics, University of Virginia, Charlottesville, VA 22904-4714, USA \\ ${ }^{4}$ Hue University College of Education, Hue, Vietnam
}

Received: 22 July 2015 / Accepted: 4 September 2015 / Published online: 24 September 2015

(c) The Author(s) 2015. This article is published with open access at Springerlink.com

\begin{abstract}
The canonical Aharonov-Bohm effect is usually studied with time-independent potentials. In this work, we investigate the Aharonov-Bohm phase acquired by a charged particle moving in time-dependent potentials. In particular, we focus on the case of a charged particle moving in the time-varying field of a plane electromagnetic wave. We work out the Aharonov-Bohm phase using both the potential (i.e. $\oint A_{\mu} \mathrm{d} x^{\mu}$ ) and the field (i.e. $\frac{1}{2} \int F_{\mu \nu} \mathrm{d} \sigma^{\mu \nu}$ ) forms of the Aharonov-Bohm phase. We give conditions in terms of the parameters of the system (frequency of the electromagnetic wave, the size of the space-time loop, amplitude of the electromagnetic wave) under which the time-varying Aharonov-Bohm effect could be observed.
\end{abstract}

\section{Introduction}

In this work, we investigate the Aharonov-Bohm phase difference picked up by charged particles that go around a closed space-time loop in the presence of the time-dependent potentials and fields of an electromagnetic plane wave.

Theoretical investigations of the Aharonov-Bohm effect $[1,2]$ generally involve time-independent potentials. The canonical example of the Aharonov-Bohm effect is that of charged particles in a two-slit experiment with an infinite solenoid, carrying a constant magnetic flux, placed between the slits. Each charged particle picks up an additional phase due to the non-zero vector potential outside the solenoid, even though the electric and magnetic fields outside the solenoid are zero. The experimental tests of the Aharonov-Bohm effect have also generally been done with time-independent fields $[3,4]$.

\footnotetext{
a e-mail: neomaxprime@mail.fresnostate.edu

be-mail: dougs@csufresno.edu

c e-mail: ay9a@virginia.edu
}

In contrast to the time-independent Aharonov-Bohm effect, there have been only a few theoretical studies of the time-dependent Aharonov-Bohm effect. Some work [5-10] has focused on a solenoid with a time-dependent magnetic flux, while other work $[11,12]$ has used an electromagnetic plane wave to obtain time-dependent potentials and fields.

On the experimental side, there are only two cases that we have found where the time-dependent Aharonov-Bohm effect was tested experimentally. The first test was an accidental experiment by Marton et al. [13] where an electron two-slit interference experiment was set up and the interference pattern was observed. However, it was later determined that the region through which the electrons traveled was contaminated with a $60 \mathrm{~Hz}$ magnetic field of unknown strength. The question in regard to the results of the accidental experiment in [13] are: "Why was the interference pattern seen at all? Why did it not shift back and forth at $60 \mathrm{~Hz}$ ?" At first, it was thought that the result of Marton et al. was evidence against the Aharonov-Bohm effect. However, two explanations have been put forward as to why the experiment in [13] saw a static interference pattern. In [14], the idea was advanced that the time-varying Aharonov-Bohm phase was compensated for by a phase shift coming from the direct $\mathbf{v} \times \mathbf{B}$ force which acted on the electrons. In [15], the explanation given for the observation of the static interference pattern in [13] was due to a cancellation between the time-varying Aharonov-Bohm phase and a phase coming from the induced electric field that accompanied the timevarying magnetic field. The second test of the time-dependent Aharonov-Bohm was the experiment in $[16,17]$. This experiment used fields from an electromagnetic wave with a frequency in the microwave region and was along the lines of the set-up suggested in [11] for testing the time-varying Aharonov-Bohm effect. This experiment was also along the lines of [12], which studied decoherence effects due to the time-varying Aharonov-Bohm phase coming from an elec- 
tromagnetic wave. The results of the experiment described in $[16,17]$ were that evidence for an Aharonov-Bohm phase from the time-varying fields and potentials was not observed - thus these results were similar to the accidental experiment of Marton et al., where the effect of the time variation was not seen in the interference pattern. The explanation of the non-observation of the time variation in the experiment [16] was that the parameters of the set-up were such that the time variation effect was too small to be seen [17]. We come to a similar conclusion from our analysis - in order to observe the time variation one must carefully choose the various parameters of the set-up: the frequency and amplitude of the electromagnetic wave, the size of the loop, the velocity of the particle, etc. In the conclusion, we give conditions under which one might see evidence of the time-varying AharonovBohm effect.

There are two points to make before we move on to our detailed analysis. First, the time-dependent Aharonov-Bohm effect is invariably a type II Aharonov-Bohm effect. The type I Aharonov-Bohm effect is when the charged particle develops a phase while moving through a region that is free of electric and magnetic fields, as in the original time-independent, infinite solenoid set-up. The type II Aharonov-Bohm effect is when the charged particle develops an Aharonov-Bohm phase, but while moving through a region of space where the fields are not zero. A typical example of a type II effect is the Aharonov-Casher effect [18], where a neutral particle with a magnetic moment moves through an electric field and, in doing so, picks up a Aharonov-Bohm-like phase. Second, the set-up we study here is a combination and generalization of the time-dependent Aharonov-Bohm effect studied in [11] and [12]. In particular for our set-up, both the electric and the magnetic Aharonov-Bohm effects are non-zero. In Ref. [11], the set-up was taken so that only the time-varying magnetic field gave an Aharonov-Bohm phase, while in Ref. [12], the set-up was such that only the time-varying electric field gave a non-zero contribution to the Aharonov-Bohm phase. Further, these two prior works calculated the Aharonov-Bohm phase in different ways: Ref. [11] used the line integral of the potentials to obtain the phase while Ref. [12] used the area integral of the fields. Here, we calculate the phase using both the line integral of the potentials and the area integral of the fields. This provides an explicit working out of Stokes' theorem in 4D. In the literature, we have found no examples of this, although there are of course plenty of explicit worked out examples of Stokes' theorem in 3D.

\section{General set-up of potentials, fields and path}

In this section, we give the set-up for the potentials, fields and path that we will use. We consider a linearly polarized plane wave traveling along the $z$-axis in either the + or direction. The covariant, four-vector potential for this is

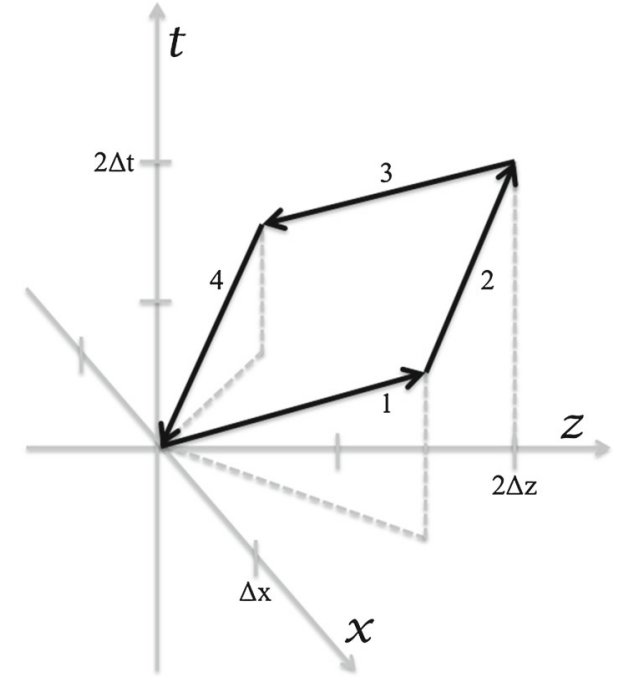

Fig. 1 The full space-time loop in $t x z$

$A_{\mu}=\left(0, A_{0} f(\omega t \pm k z), 0,0\right)$,

where $A_{0}$ is the amplitude and $\omega, k$ are the frequency and wave number for the wave. The $(+)$ sign is a wave traveling in the $-z$ direction and the $(-)$ is a wave traveling in the $+z$ direction. The electric and magnetic fields can be obtained from (1) using $F_{\mu \nu}=\partial_{\mu} A_{\nu}-\partial_{\nu} A_{\mu}$ with the result

$F_{01}=E_{x}=A_{0} \frac{\omega}{c} f^{\prime}(\omega t \pm k z) ;$

$F_{13}=B_{y}=\mp A_{0} k f^{\prime}(\omega t \pm k z)$,

where a prime means a derivative with respect to the argument of $f$, namely $\zeta_{ \pm}=\omega t \pm k z$. The electromagnetic wave in (2) is polarized in the $x$ direction. We could consider a more complicated wave traveling in the $z$-direction, with the electric and magnetic fields having components in both $x$ and $y$ directions. The vector potential for such wave would have the form $A_{\mu}=$ $\left(0, A_{0} f\left(\omega t \pm k z+\varphi_{1}\right), B_{0} f\left(\omega t \pm k z+\varphi_{2}\right), 0\right)$, where $A_{0}$, $B_{0}$ are amplitudes and $\varphi_{1}, \varphi_{2}$ are phases. For our purposes, the four-potential in (1) is sufficient since for the closed loop path that we pick, with no motion of the particles in the $y$ direction, only the $x$ component of the four-vector potential will contribute. If we had picked our loop to be in the $y z$ plane, then it would be a vector potential of the form $A_{\mu}=\left(0,0, B_{0} f(\omega t \pm k z), 0\right)$, which would give a non-zero contribution.

The closed space-time path that we chose in evaluating the loop integral $\oint A_{\mu} \mathrm{d} x^{\mu}$ is shown fully in Fig. 1. The projections in the $t x$ and $z x$ planes are shown, respectively, in Figs. 2 and 3. In the experiment, the charged particles would travel both paths 1 and 2, and 3 and 4, in the forward timedirection, i.e. the charged particles would leave the origin and travel forward along 1 and 2 and also forward along 3 and 4, 


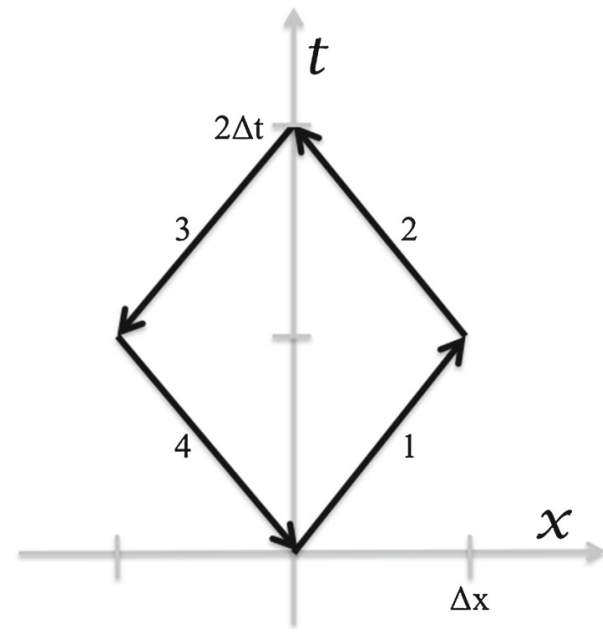

Fig. 2 The projection of the space-time loop in $t x$ plane. This also represents the upright diamond which we will discuss in the next section

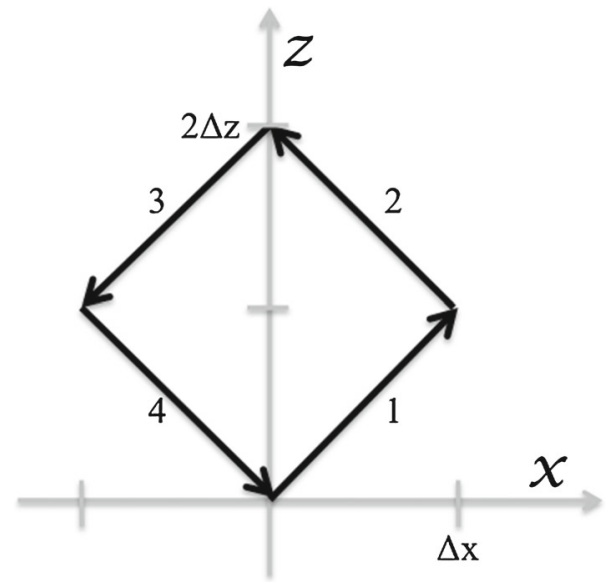

Fig. 3 The projection of the space-time loop in xz plane

with each picking up some different Aharonov-Bohm phase along these paths. To take the phase difference between path 1 and 2 versus path 3 and 4 , one puts a negative sign in front of the integral along path 3 and 4 (or as well one could put the negative sign in front of the integrals along path 1 and 2) thus reversing the direction of the space-time loop in Fig. 1. In this way, one ends with a closed space-time loop integral. This is what is done in the usual static Aharonov-Bohm analysis, but with purely spatial loop integrals.

The paths start from the origin, $x=z=t=0$, and then travel with velocity $\mathbf{v}=\left( \pm v_{x}, 0, v_{z}\right)$, for a time $\Delta t$, to the two spatial points $( \pm \Delta x, 0, \Delta z)$. This gives paths 1 and 4 . From these two points, one traces back to the point $(0,0,2 \Delta z)$ in a time $\Delta t$ (this means a total time of $2 \Delta t)$; this gives paths 2 and 3 . Note, the total time from the origin to $(0,0,2 \Delta z)$ is $2 \Delta t$, so $\Delta t=\frac{\Delta z}{v_{z}}=\frac{\Delta x}{v_{x}}$. Now, for the explicit evaluation of $\oint A_{\mu} \mathrm{d} x^{\mu}$ in the next section, we need to give the equations describing each path:
Path $1(0<t<\Delta t): t=\frac{x}{v_{x}}=\frac{z}{v_{z}}$,

Path $2(\Delta t<t<2 \Delta t): t=-\frac{x}{v_{x}}+2 \Delta t=\frac{z}{v_{z}}$,

Path $3(\Delta t<t<2 \Delta t): t=\frac{x}{v_{x}}+2 \Delta t=\frac{z}{v_{z}}$,

Path $4(0<t<\Delta t): t=-\frac{x}{v_{x}}=\frac{z}{v_{z}}$.

The magnitude of the velocity along any path is $v=$ $\sqrt{v_{x}^{2}+v_{z}^{2}} \leq c$. For the projections of the full space-time path from Fig. 1 into the $t x$ plane in Fig. 2, the slopes of the paths are greater than 1 since $v_{x}, v_{z}<c$.

For the Aharonov-Bohm effect with a plane wave background, both the electric and the magnetic Aharonov-Bohm effects can be non-trivial at the same time. For the usual static magnetic Aharonov-Bohm effect, the line integral of the 3-vector potential, $\mathbf{A}$, is related to the surface area of the magnetic field via Stokes' theorem in 3D, $\oint \mathbf{A} \cdot \mathrm{d} \mathbf{x}=\int \mathbf{B} \cdot \mathrm{d} \mathbf{a}$. In order for this magnetic Aharonov-Bohm phase to be non-zero, the magnetic field, $\mathbf{B}$, must have a component along the area normal direction, da. For our space-time area, the spatial projection is in the $x z$ plane, which therefore has a spatial area in the $y$-direction. This then gives a non-zero contribution for the magnetic field of the plane wave since $\mathbf{B} \propto \hat{\mathbf{y}}$. For the usual static electric AharonovBohm effect, it is the time integral of the scalar potential, $\phi$, which is important (i.e. $\int \phi \mathrm{d} t$ ). Using $\mathbf{E}=-\nabla \phi$, one finds that the electric Aharonov-Bohm phase can be written as $\int \phi \mathrm{d} t=-\int \mathbf{E} \cdot \mathrm{d} \mathbf{x} \mathrm{d} t$. Note that the spatial area of the magnetic case, $\mathrm{d} \mathbf{a}$, is replaced by a space-time area $\mathrm{d} \mathbf{x} \mathrm{d} t$. In order to get an electric contribution to the Aharonov-Bohm phase, the electric field must have a component along the direction of the path.

For the wave traveling in the $z$-direction, and for the area of the loop which has a projection in the $x z$ plane, both the magnetic field in the $y$-direction and the electric field in the $x$ direction will give non-zero contributions to the time-varying Aharonov-Bohm phase. In the case studied in [12], the wave was taken to be traveling in the $y$-direction, the electric field was polarized in the $z$-direction, and the magnetic field was in the $x$-direction. The loop chosen in [12] was also in the $x z$ plane, so, in that situation, there was only a non-zero electric Aharonov-Bohm phase; the magnetic Aharonov-Bohm phase was zero. In our set-up both the magnetic and electric Ahronov-Bohm phases play a role.

\section{Aharonov-Bohm phase via the potentials}

Using the above set-up, we now calculate $\oint A_{\mu} \mathrm{d} x^{\mu}$, which gives the Aharonov-Bohm phase when multiplied by $\frac{e}{\hbar c}$. We will calculate the phase picked up along the four path lengths 
given in Fig. 1 and then add them. The electromagnetic wave has a frequency and wave number given by $\omega$ and $k$, which satisfy $\frac{\omega}{k}=c$. The velocity on any given leg of the path in Fig. 1 is $\mathbf{v}=\left( \pm v_{x}, 0, v_{z}\right)$.

In carrying out the calculation for the loop integral for the case in Fig. 1, we will first calculate $\oint A_{\mu} \mathrm{d} x^{\mu}$ in the frame where $v_{z}=0$; this is the "upright diamond" loop of Fig. 2 . Then, to obtain the more general case, we will boost the result of the upright diamond so that the particle develops a velocity, $v_{z}$, in the $+z$ direction.

The "upright diamond" For the upright diamond in Fig. 2, we have $z=0$ and $\mathbf{v}=\left( \pm v_{0}, 0,0\right)$, and we take the wave number and frequency as $k_{0}$ and $\omega_{0}$, respectively. The four paths are parametrized as:

Path $1(0<t<\Delta t): t=\frac{x}{v_{0}}$,

Path $2(\Delta t<t<2 \Delta t): t=-\frac{x}{v_{0}}+2 \Delta t$,

Path $3(\Delta t<t<2 \Delta t): t=\frac{x}{v_{0}}+2 \Delta t$,

Path $4(0<t<\Delta t): t=-\frac{x}{v_{0}}$.

Along each path, since $z=0, f\left(\omega_{0} t \pm k_{0} z\right)$ simplifies to $f\left(\omega_{0} t\right)$. For path 1 , from (7), we find

$$
\begin{aligned}
\int_{1} A_{\mu} \mathrm{d} x^{\mu} & =A_{0} \int_{0}^{\Delta x} f\left(\frac{k_{0}}{\beta_{0}} x\right) \mathrm{d} x=\frac{A_{0} \beta_{0}}{k_{0}}\left[F\left(\Delta \zeta_{0}\right)-F(0)\right] \\
& =A_{0} \Delta x\langle f(\zeta)\rangle_{\mathrm{I}}
\end{aligned}
$$

where $\beta_{0}=v_{0} / c, c k_{0}=\omega_{0}$, and $F(\zeta)=\int f(\zeta) \mathrm{d} \zeta$ is the integral function of $f . \Delta \zeta_{0}$ is the phase shift up to the halfway point,

$\Delta \zeta_{0}=\frac{k_{0}}{\beta_{0}} \Delta x=\omega_{0} \Delta t$.

In the final expression in (11) we have written the result in terms of $\langle f(\zeta)\rangle_{\mathrm{I}} \equiv \frac{1}{\Delta \zeta_{0}} \int_{0}^{\Delta \zeta_{0}} f(\zeta) d \zeta$, which is the average of $f(\zeta)$ in the interval $\mathrm{I}=\left\{\zeta: 0<\zeta<\Delta \zeta_{0}\right\}$.

For path 2, we have, from (8),

$$
\begin{aligned}
& \int_{2} A_{\mu} \mathrm{d} x^{\mu}=A_{0} \int_{\Delta x}^{0} f\left(-\frac{k_{0}}{\beta_{0}} x+2 \omega_{0} \Delta t\right) \mathrm{d} x \\
& =-\frac{A_{0} \beta_{0}}{k_{0}}\left[F\left(2 \omega_{0} \Delta t\right)-F\left(-\frac{k_{0}}{\beta_{0}} \Delta x+2 \omega_{0} \Delta t\right)\right] \\
& =-\frac{A_{0} \beta_{0}}{k_{0}}\left[F\left(2 \Delta \zeta_{0}\right)-F\left(\Delta \zeta_{0}\right)\right]=-A_{0} \Delta x\langle f(\zeta)\rangle_{\mathrm{II}},
\end{aligned}
$$

where $\langle f(\zeta)\rangle_{\mathrm{II}}$ is the average of $f(\zeta)$ over the interval $\mathrm{II}=$ $\left\{\zeta: \Delta \zeta_{0}<\zeta<2 \Delta \zeta_{0}\right\}$.

In a similar manner, for path 3 and path 4 , one finds

$$
\int_{3} A_{\mu} \mathrm{d} x^{\mu}=\int_{2} A_{\mu} \mathrm{d} x^{\mu} ; \quad \int_{4} A_{\mu} \mathrm{d} x^{\mu}=\int_{1} A_{\mu} \mathrm{d} x^{\mu} .
$$

Altogether, the upright diamond loop integral is

$$
\begin{aligned}
\oint A_{\mu} \mathrm{d} x^{\mu}= & \int_{1} A_{\mu} \mathrm{d} x^{\mu}+\int_{2} A_{\mu} \mathrm{d} x^{\mu}+\int_{3} A_{\mu} \mathrm{d} x^{\mu} \\
& +\int_{4} A_{\mu} \mathrm{d} x^{\mu}=2 \frac{A_{0} \beta_{0}}{k_{0}}\left(\left[F\left(\Delta \zeta_{0}\right)-F(0)\right]\right. \\
& \left.-\left[F\left(2 \Delta \zeta_{0}\right)-F\left(\Delta \zeta_{0}\right)\right]\right) \\
= & 2 \frac{A_{0} \beta_{0}}{k_{0}}\left(2 F\left(\Delta \zeta_{0}\right)-F(0)-F\left(2 \Delta \zeta_{0}\right)\right),
\end{aligned}
$$

or in terms of the averages of $f(\zeta)$,

$\oint A_{\mu} \mathrm{d} x^{\mu}=2 A_{0} \Delta x\left(\langle f(\zeta)\rangle_{\mathrm{I}}-\langle f(\zeta)\rangle_{\mathrm{II}}\right)$.

The loop integral is zero if the average of the vector potential in the first half (I) and the second half (II) are the same. Boost along $z$-direction In order to evaluate the loop integral for $v_{z} \neq 0$, we now boost the frame along the $z$-direction by $\beta$. This gives the particle a velocity in the $z$ direction, and changes the upright diamond of Fig. 2 to the tilted diamond of Fig. 1. In Eq. (15), the only parameters that change are the wave number $k_{0}$ and the $x$-component of velocity, $\beta_{0} . \Delta \zeta_{0}$ also transforms due to $k_{0}$ and $\beta_{0}$ changing. The parameters $\Delta x$ and $A_{0}$ do not change since they are perpendicular to the boost direction. After the boost,

$\beta_{0} \rightarrow \beta_{x}=\sqrt{1-\beta^{2}} \beta_{0}=\beta_{0} / \gamma ; \quad k_{0} \rightarrow k=\sqrt{\frac{1 \pm \beta}{1 \mp \beta}} k_{0}$.

In Eq. (15), only the combination $\frac{k_{0}}{\beta_{0}}$ shows up. Using (17) we find

$\frac{k_{0}}{\beta_{0}} \rightarrow \sqrt{\frac{1 \mp \beta}{1 \pm \beta}} k \cdot \frac{1}{\gamma \beta_{x}}=(1 \mp \beta) \frac{k}{\beta_{x}}$

Setting $\beta=-\beta_{z}=-v_{z} / c$, we get the final result,

$$
\begin{aligned}
\oint A_{\mu} \mathrm{d} x^{\mu}= & \frac{2 A_{0}}{k} \frac{\beta_{x}}{1 \pm \beta_{z}}\left[2 F\left(\frac{1 \pm \beta_{z}}{\beta_{x}} k \Delta x\right)-F(0)\right. \\
& \left.-F\left(2 \frac{1 \pm \beta_{z}}{\beta_{x}} k \Delta x\right)\right] \\
= & \frac{2 A_{0}}{k^{\prime}}\left[2 F\left(k^{\prime} \Delta x\right)-F(0)-F\left(2 k^{\prime} \Delta x\right)\right] \\
= & \frac{2 A_{0}}{k^{\prime}}[2 F(\Delta \zeta)-F(0)-F(2 \Delta \zeta)],
\end{aligned}
$$

where $k^{\prime}$ is defined as

$k^{\prime}=\frac{1 \pm \beta_{z}}{\beta_{x}} k$,

and $\Delta \zeta$ is the phase shift to the half-way point,

$\Delta \zeta=k^{\prime} \Delta x=\omega\left(1 \pm \beta_{z}\right) \Delta t=\omega \Delta t \pm k \Delta z$. 
The last line of Eq. (19) is the main result of this section and we want to make some comments/remarks about its physical meaning. First, we can look at an expansion of the function $F(\eta)=F(0)+\eta F^{\prime}(0)+\frac{1}{2} \eta^{2} F^{\prime \prime}(0)+\cdots$, where $\eta=\Delta \zeta=k^{\prime} \Delta x$ or $\eta=2 \Delta \zeta=2 k^{\prime} \Delta x$, depending on which term in (19) one is expanding. Using this, one finds that (19) becomes

$$
\oint A_{\mu} \mathrm{d} x^{\mu}=-2 A_{0} k^{\prime} \Delta x^{2} F^{\prime \prime}(0)+\mathcal{O}\left((\Delta \zeta)^{3}\right) .
$$

Thus, to first order in $\Delta \zeta$, the time-varying AharonovBohm phase vanishes. This result can be compared to the result in $[9,10]$, where the authors found that for a solenoid with a time-varying flux, the time-dependent part of the Aharonov-Bohm phase vanishes to first order as well.

Next, if we take the particle speed to be much less than the speed of light, $v=\sqrt{v_{x}^{2}+v_{z}^{2}} \ll c$, then, from (20), $k^{\prime} \approx k / \beta_{x}$. Hence the condition $k^{\prime} \Delta x \ll 1$ is identical to $\Delta x / \beta_{x} \ll \lambda$, or equivalently $\Delta t \ll T=\frac{2 \pi}{\omega}$, where $\lambda$ and $T$ are the wavelength and the period of the electromagnetic wave. Since the approximate vanishing of $\oint A_{\mu} \mathrm{d} x^{\mu}$ requires $k^{\prime} \Delta x \ll 1$, the condition above imposed on the wavelength or the period of the electromagnetic wave is a condition for the approximate vanishing of the loop integral. At the end of the next section, we will comment more on the use of the result in (22) to determine when the time-dependent Aharonov-Bohm effect is important/observable.

One final question we want to address: "under what conditions does $\oint A_{\mu} \mathrm{d} x^{\mu}$ vanish exactly". According to (19) and (22), if $k^{\prime} \rightarrow 0$, the loop integral vanishes, which in turn means $\beta_{z} \rightarrow \mp 1$ according to (20). Thus, if the particle traverses the loop at close to $c$ and moves in the same direction as the electromagnetic wave (in this case the $z$ direction) the time-dependent Aharonov-Bohm phase will vanish. This was the same conclusion reached for the timedependent, non-Abelian Aharonov-Bohm effect [19] using the time-dependent, non-Abelian plane waves of Coleman [20]. However, in the present case, due to the constraint $\sqrt{\beta_{x}^{2}+\beta_{z}^{2}} \leq 1, \beta_{z} \rightarrow 1$ means $\beta_{x} \rightarrow 0$, the $x$-component of the velocity is vanishingly small, and that the diamond shaped loop becomes elongated in the $z$ direction since the ratio of the lengths goes as $\Delta x / \Delta z \rightarrow 0$.

There is another way that the loop integral vanishes exactly. Rewriting (19) in terms of the original function $f(\omega t \pm k z)$, we have

$\oint A_{\mu} \mathrm{d} x^{\mu}=2 A_{0} \Delta x\left(\langle f(\zeta)\rangle_{\mathrm{I}}-\langle f(\zeta)\rangle_{\mathrm{II}}\right)$,

where the notation is similar to Eq. (16) and with the intervals defined as

$\mathrm{I}=\{\zeta: 0<\zeta<\Delta \zeta\} ; \quad \mathrm{II}=\{\zeta: \Delta \zeta<\zeta<2 \Delta \zeta\}$, where $\Delta \zeta=\omega \Delta t \pm k \Delta z$, is the phase change to the half-way point. This means that if the average of the vector potential experienced by the particle in the first half, $0<t<\Delta t$, is the same as the average of that experienced in the latter half, $\Delta t<t<2 \Delta t$, then the loop integral vanishes. From (19) this happens if the integrand function, $F(\zeta)$, is linear with respect to $\zeta$. This is another way of giving the result from (22) that $\oint A_{\mu} \mathrm{d} x^{\mu}$ vanishes to zeroth and first order in $F(\zeta)$.

\section{Aharonov-Bohm phase via the fields}

In this section, we work out the Aharonov-Bohm phase using the electric and magnetic fields and taking the "area" integrals. This is a 4D example of the usual 3D Stokes' theorem, where one finds $\oint \mathbf{A} \cdot \mathrm{d} \mathbf{x}=\int \mathbf{B} \cdot \mathrm{d} \mathbf{a}$. In the 4D case, we want $\oint A_{\mu} \mathrm{d} x^{\mu}=\frac{1}{2} \int F_{\mu \nu} \mathrm{d} x^{\mu} \wedge \mathrm{d} x^{\nu}$, where we have written the $4 \mathrm{D}$ area $\mathrm{d} \sigma^{\mu \nu} \rightarrow \mathrm{d} x^{\mu} \wedge \mathrm{d} x^{\nu}$, using the antisymmetric wedge product. Concise details of this notation can be found in $[21,22]$. Taking the components of the electric and magnetic fields given in (2), we have

$$
\begin{aligned}
\int F= & \frac{1}{2} \int F_{\mu \nu} \mathrm{d} x^{\mu} \wedge \mathrm{d} x^{\nu}=-\iint E_{x} \mathrm{~d} x \wedge c \mathrm{~d} t \\
& -\iint B_{y} \mathrm{~d} z \wedge \mathrm{d} x .
\end{aligned}
$$

In (25) we have written things in three vector notation with $E_{x}=F_{01}$ and $B_{y}=F_{13}$. The first term is a space-time area integral of the electric field, $E_{x}$, and the second term is a purely spatial area integral of the magnetic field, $B_{y}$. As in Sect. 3 we will do the "upright diamond" area and show that this is equivalent to the loop integral of the "upright diamond" from (15). Then by boosting in the $z$ direction, one can obtain the area integral for the general surface from Fig. 1. For the upright diamond loop one has a surface only in the $x t$-plane (see Fig. 2), and therefore only an electric contribution:

$\int_{\text {upright }} F=-\iint E_{x} \mathrm{~d} x \wedge c \mathrm{~d} t$

We will split the upright diamond into a left triangle and a right triangle, evaluate these separately and then sum them up:

$\int_{\text {upright }} F=-\left(\int_{\text {left }}+\int_{\text {right }}\right) E_{x} \mathrm{~d} x \wedge c \mathrm{~d} t$. 
For the upright diamond, $z=0$ and $k, \omega \rightarrow k_{0}, \omega_{0}$, so from (2) we have $E_{x}=F_{01}=A_{0} \frac{\omega}{c} f^{\prime}(\omega t \pm k z) \rightarrow$ $A_{0} \frac{\omega_{0}}{c} f^{\prime}\left(\omega_{0} t\right)$. With this the left half of the surface integral is

$$
\begin{aligned}
& -\iint_{\text {left }} E_{x} \mathrm{~d} x \wedge c \mathrm{~d} t=-\int_{-\Delta x}^{0} \int_{-x / v_{0}}^{2 \Delta t+x / v_{0}} A_{0} \omega_{0} f^{\prime}\left(\omega_{0} t\right) \mathrm{d} x \mathrm{~d} t \\
& =-\int_{-\Delta x}^{0} A_{0}\left[f\left(2 \omega_{0} \Delta t+\frac{\omega_{0} x}{v_{0}}\right)-f\left(-\frac{\omega_{0} x}{v_{0}}\right)\right] \mathrm{d} x \\
& =\frac{A_{0} \beta_{0}}{k_{0}}\left(2 F\left(\Delta \zeta_{0}\right)-F(0)-F\left(2 \Delta \zeta_{0}\right)\right)
\end{aligned}
$$

where $\Delta \zeta_{0}=\omega_{0} \Delta t=\left(k_{0} / \beta_{0}\right) \Delta x$.

Likewise, for the right half of the upright diamond, we have

$$
\begin{aligned}
& -\iint_{\text {right }} E_{x} \mathrm{~d} x \wedge c \mathrm{~d} t=-\int_{0}^{\Delta x} \int_{x / v_{0}}^{2 \Delta t-x / v_{0}} A_{0} \omega_{0} f^{\prime}\left(\omega_{0} t\right) \mathrm{d} x \mathrm{~d} t \\
& =-\int_{0}^{\Delta x} A_{0}\left[f\left(2 \omega_{0} \Delta t-\frac{\omega_{0} x}{v_{0}}\right)-f\left(\frac{\omega_{0} x}{v_{0}}\right)\right] \mathrm{d} x \\
& =\frac{A_{0} \beta_{0}}{k_{0}}\left(2 F\left(\Delta \zeta_{0}\right)-F(0)-F\left(2 \Delta \zeta_{0}\right)\right) .
\end{aligned}
$$

Adding (28) and (29) gives the final result for the upright diamond of

$$
\int_{\text {upright }} F=2 \frac{A_{0} \beta_{0}}{k_{0}}\left[2 F\left(\Delta \zeta_{0}\right)-F(0)-F\left(2 \Delta \zeta_{0}\right)\right] .
$$

This is the identical result for the upright diamond loop integral of Eq. (15), which confirms the 4D Stokes' theorem. To get the case with a general velocity in the $z$ direction, we can boost the result from Eq. (30), as we did in Sect. 3, to obtain the final result of Eq. (19).

The upright diamond area integral is solely due to electric field. However, after the boost, it gets a magnetic contribution. The ratio of the electric and magnetic contributions depends on the magnitude of the boost $\beta_{z}$. One can calculate the electric and magnetic contributions separately. Denoting the Aharonov-Bohm phase due to electric and magnetic field as $\Delta \phi_{E}$ and $\Delta \phi_{B}$, respectively, one can derive (see the Appendix)

$\frac{\Delta \phi_{B}}{\Delta \phi_{E}}=\frac{\int F_{31} \mathrm{~d} z \wedge \mathrm{d} x}{\int F_{10} \mathrm{~d} x \wedge c \mathrm{~d} t}= \pm \beta_{z}$.

The ratio only depends on $\beta_{z}$. At any speed less than $c$, the electric contribution is larger. This is the reverse of what was found in Ref. [7], under different conditions. In [7], the timedependent system was an infinite solenoid with a magnetic flux which was pulsed on over a short time. In this case, the magnetic Aharonov-Bohm phase was dominant over the phase shift due to the electric Aharonov-Bohm effect. In $[9,10]$, the case of an infinite solenoid with a slowly varying flux was considered. To linear order it was found that the electric and magnetic Aharonov-Bohm phase shifts were of equal magnitude but opposite sign and thus canceled.
For the case of the electromagnetic wave, the magnetic contribution becomes equal to the electric contribution only when the particles travel at the speed of light. When they travel at $c$ against the waves, the two contributions add up; when they travel at $c$ along the waves - riding on the waves the two contributions cancel completely and result in a zero time-dependent Aharonov-Bohm phase, as derived in Sect. 3. In both cases, due to the relativistic constraint, $\beta_{x} \rightarrow 0$ as $\beta_{z} \rightarrow 1$. This behavior is different from the case of the solenoid, where reversing the magnetic field of the solenoid also reverses the direction of the electric field. Thus, in the case of the solenoid, one has the same result whether the magnetic field points along the $+z$ or $-z$ direction. In contrast, reversing the direction of the electromagnetic wave only reverses the direction of one of the fields, so, in one case, the electric and magnetic contributions add up, while in the other case, the electric and magnetic contributions tend to cancel.

Using either the area integral of the fields or the line integral of the potentials, the Aharonov-Bohm phase picked up in going around the space-time loop in Fig. 1 is

$$
\left(\frac{e}{\hbar c}\right)\left(\frac{2 A_{0}}{k^{\prime}}\right)\left[2 F\left(k^{\prime} \Delta x\right)-F\left(2 k^{\prime} \Delta x\right)-F(0)\right] .
$$

We now recall the expansion of the term in square bracket from (19), in terms of $k^{\prime} \Delta x=\Delta \zeta$, and apply this to (32) to give the Aharonov-Bohm phase up to $\mathcal{O}\left(k^{\prime} \Delta x\right)^{3}$ as

$$
\begin{aligned}
\frac{1}{2}\left(\frac{e}{\hbar c}\right) \int F_{\mu \nu} \mathrm{d} x^{\mu} \wedge \mathrm{d} x^{\nu} & =\left(\frac{e}{\hbar c}\right) \oint A_{\mu} \mathrm{d} x^{\mu} \\
& =-2\left(\frac{e}{\hbar c}\right) A_{0} k^{\prime} \Delta x^{2} F^{\prime \prime}(0)
\end{aligned}
$$

From the above equation, we can see that in order for the time-dependent Aharonov-Bohm effect to be observable, one wants to have

$$
\left(\frac{e}{\hbar c}\right) A_{0} k^{\prime} \Delta x^{2} \sim \mathcal{O}(1)
$$

In writing (34) we have ignored the factor of -2 and assumed that $F^{\prime \prime}(0) \sim \mathcal{O}(1)$, which would be the case if $f\left(\zeta_{ \pm}\right)$and $F\left(\zeta_{ \pm}\right)$were sinusoidal.

We now investigate a few different scenarios for the condition in (34). In regard to the spatial size of our loop, we follow [12] and estimate that $\Delta x \sim 100 \mu \mathrm{m}$. We will take the speed of our particle to be non-relativistic with $v \sim 10^{6} \frac{\mathrm{m}}{\mathrm{s}}$. Thus, from (20), we have $k^{\prime} \approx k \frac{c}{v}=\frac{\omega}{v}$. Under these assumptions, the condition in (34) has two free parameters: $A_{0}$ and $\omega$. Combining all this, we find that the condition in (34) becomes

$$
A_{0} \omega \sim 10^{8} \mathrm{~J} /(\mathrm{C} \mathrm{s})
$$


Conditions (34) and (35) can be used to determine how to pick $A_{0}$ and/or $\omega$ in order to see the time-dependent Aharonov-Bohm effect experimentally. From (35), we find that one should pick $A_{0} \omega$ to be of order $10^{8}$. However, if one picks $A_{0}$ and/or $\omega$ to be too large, the electrons will experience a direct force which could then overwhelm the Aharonov-Bohm effect. For example, one could consider soft $x$-ray frequencies of $\omega \sim 10^{17} \mathrm{~Hz}$, which would only require an amplitude of $A_{0} \sim 10^{-9} \frac{\mathrm{J}}{\mathrm{C}}$. However, the $x$-ray photons could scatter the electrons significantly. Whether this would be an issue would depend on the photon number density, which is related to the energy density, $\rho_{E \& M} \propto A_{0}^{2} \omega^{2}$. Alternatively, one could start by picking a smaller $\omega$ and therefore a larger $A_{0}$. One does not want to make $\omega$ too small since this would imply a large wavelength $\lambda=2 \pi / k$ and the large wavelength limit tends to force the time-dependent Aharonov-Bohm phase to zero. As a crude estimate, we might require that the wavelength of the electromagnetic wave be one order of magnitude smaller than the loop size. Since we have set the scale for the loop size at $\Delta x \sim 100 \mu \mathrm{m}$, as in [12], this estimate implies we should take $\lambda \sim 10 \mu \mathrm{m}$. This yields a frequency of $\omega \sim 10^{14} \mathrm{~Hz}$, which is in the infrared/optical part of the spectrum. For this choice of $\omega$, Eq. (35) implies that one would need $A_{0} \sim 10^{-6} \frac{\mathrm{J}}{\mathrm{C}}$. The above discussion is by no means exhaustive, but it is merely to show the interrelatedness of the system parameters, $A_{0}$ and $\omega$ (and in more general situations $\Delta x$ and $v$ ), in determining when the time-dependent Aharonov-Bohm phase would be observable. Some of the same conclusion were reached in [17].

\section{Conclusions and summary}

In this article, we have investigated the time-dependent Aharonov-Bohm phase of a charged particle traveling around a closed space-time loop, given in Figs. 1, 2 and 3, in the presence of a time-varying electromagnetic field, given by a plane wave traveling in the $\pm z$ direction and with a polarization in the $x$ direction. Our work is a generalization of earlier work, $[11,12]$, in that we consider both the electric and the magnetic Aharonov-Bohm effects, and thus we are able to see the interplay between the two. In addition, we calculated the time-varying Aharonov-Bohm phase both in terms of the path integral of the vector potential, $\oint A_{\mu} \mathrm{d} x^{\mu}$, and in terms of the area integral of the fields, $\frac{1}{2} \int F_{\mu \nu} \mathrm{d} x^{\mu} \wedge \mathrm{d} x^{\nu}$.

The overall conclusion of our calculation of the AharonovBohm phase for the time-varying fields of an electromagnetic plane wave is that this phase vanishes under a broad range of conditions. Thus one must carefully choose the parameters of the electromagnetic wave and of the loop that the electrons traverse in order to see the effect. This is similar to the conclusion of the work in $[16,17]$ where the time-varying
Aharonov-Bohm phase was searched for experimentally but not observed. First, from (23), one finds that the time-varying Aharonov-Bohm phase vanishes if the average of the wave form over the first half of the loop equals the average over the second half of the loop i.e. $\langle f(\zeta)\rangle_{\mathrm{I}}=\langle f(\zeta)\rangle_{\mathrm{II}}$. Second, from (33), we showed that expanding the time-varying AharonovBohm phase, to lowest order, gave a phase with a magnitude of $\left(\frac{e}{\hbar c}\right) A_{0} k^{\prime} \Delta x^{2}$. In this expansion, only the second order term in $k^{\prime} \Delta x$ remained; the zeroth and first order terms vanished. Third, in the long wavelength limit (i.e. $k, k^{\prime} \rightarrow 0$ ), one finds that the functional part of the Aharonov-Bohm phase from (32) vanishes i.e. $\left[2 F\left(k^{\prime} \Delta x\right)-F\left(2 k^{\prime} \Delta x\right)-F(0)\right] \rightarrow 0$. This occurs when the velocity of the particle traversing the loop moves completely in the $z$ direction at the speed of light, $\beta_{z}=1$. In this case the particle "rides along" with the electromagnetic wave. This smallness of the time-varying Aharonov-Bohm phase is in accord with earlier results. In Refs. $[9,10,15]$, it was found that the time-varying part of the Aharonov-Bohm phase shift vanished under the assumption that the time variation of the flux in the solenoid was slow compared to the time it took the charged particles to traverse the loop.

In order to see the time-varying Aharonov-Bohm effect, we found a general condition, Eq. (34), which needed to be satisfied. This condition depended on the wave amplitude, frequency, particle velocity and size of the loop $-A_{0}, \omega, v$, and $\Delta x$. By making some reasonable choices (i.e. $v \sim 10^{6} \frac{\mathrm{m}}{\mathrm{s}}$ and $\Delta x \sim 100 \mu \mathrm{m}$ ) we arrived at the more specific condition $A_{0} \omega \sim 10^{8} \mathrm{~J} /(\mathrm{C} \mathrm{s})$. One could easily satisfy this condition by making either $A_{0}$ and/or $\omega$ large enough. However, making $A_{0}$ large increases the strength of the electric field and making $\omega$ large increased the energy of the photons of the time-varying field. Both of these effects would tend to put forces on (i.e. scatter) the charged particles, destroying the pure Aharonov-Bohm effect, which is a phase shift not due to direct forces. To observe the Aharonov-Bohm effect, one wants to reduce as far as possible the forces/scattering, which means making $A_{0}$ and $\omega$ small. There is some trade off in terms of the magnitude of $A_{0}$ and $\omega$ - one needs the product of $A_{0}$ and $\omega$ to be large enough to see the time-varying Aharonov-Bohm effect, but if one makes either of these two parameters too large the electron will experience significant direct forces, which will spoil the Aharonov-Bohm interference pattern.

Note added in proof After this paper was accepted we learned of Ref. [23] which deals with some experimental issues of the AharonovBohm effect connected with the present work.

Acknowledgments DS is supported by a 2015-2016 Fulbright Scholars Grant to Brazil. DS wishes to thank the ICTP-SAIFR in São Paulo for it hospitality. DS also acknowledges support by a Grant (number 1626/GF3) in Fundamental Research in Natural Sciences by the Science Committee of the Ministry of Education and Science of Kazakhstan. 
Open Access This article is distributed under the terms of the Creative Commons Attribution 4.0 International License (http://creativecomm ons.org/licenses/by/4.0/), which permits unrestricted use, distribution, and reproduction in any medium, provided you give appropriate credit to the original author(s) and the source, provide a link to the Creative Commons license, and indicate if changes were made.

Funded by SCOAP ${ }^{3}$.

\section{Appendix I}

In this appendix, we derive the ratio $\Delta \phi_{B} / \Delta \phi_{E}$ given in (31). The total phase shift for the tilted diamond, given in (19), can be split into an electric and magnetic contribution as

$$
\Delta \phi=\Delta \phi_{E}+\Delta \phi_{B}=\frac{2 A_{0}}{k^{\prime}}[2 F(\Delta \zeta)-F(0)-F(2 \Delta \zeta)]
$$

To find the ratio of the magnetic to electric contributions, we just need to find $\Delta \phi_{E}$ for the "tilted diamond" and then use this in (36) to obtain $\Delta \phi_{B}$. As in Sect. 4, we calculate the electric part of the tilted diamond by calculating the left and right sides and adding these together. Using (2), the electric contribution for the left side of the tilted diamond is

$$
\begin{aligned}
\left(\Delta \phi_{E}\right)_{\text {left }}= & -\iint_{\text {left }} E_{x} \mathrm{~d} x \wedge c \mathrm{~d} t \\
= & -A_{0} \frac{\omega}{c} \int_{-\Delta x}^{0} \mathrm{~d} x\left(\int_{-x / v_{x}}^{2 \Delta t+x / v_{x}} f^{\prime}(\omega t \pm k z) c \mathrm{~d} t\right) \\
= & -A_{0} \frac{\omega}{\omega^{\prime}} \int_{-\Delta x}^{0}\left[f\left(2 \omega^{\prime} \Delta t+\omega^{\prime} x / v_{x}\right)\right. \\
& \left.-f\left(-\omega^{\prime} x / v_{x}\right)\right] \mathrm{d} x
\end{aligned}
$$

In the above, we have replaced $z=v_{z} t$ using (3) and defined $\omega^{\prime}=\left(\omega \pm k v_{z}\right)=\omega\left(1 \pm \beta_{z}\right)$. Next, performing the $x$ integration in (37), we find

$$
\begin{aligned}
\left(\Delta \phi_{E}\right)_{\mathrm{left}}= & -A_{0} \frac{\omega v_{x}}{\left(\omega^{\prime}\right)^{2}}\left[F\left(2 \omega^{\prime} \Delta t\right)-F\left(\omega^{\prime} \Delta t\right)\right. \\
& \left.+F(0)-F\left(\omega^{\prime} \Delta t\right)\right]=A_{0} \frac{\beta_{x}}{k\left(1 \pm \beta_{z}\right)^{2}} \\
& \times\left[2 F\left(\omega^{\prime} \Delta t\right)-F\left(2 \omega^{\prime} \Delta t\right)-F(0)\right] \\
= & A_{0} \frac{1}{k^{\prime}\left(1 \pm \beta_{z}\right)}[2 F(\Delta \zeta)-F(2 \Delta \zeta)-F(0)],
\end{aligned}
$$

where we have used $k=\frac{\beta_{x}}{1 \pm \beta_{z}} k^{\prime}$ from (20) and $\Delta \zeta=\omega^{\prime} \Delta t$ from (21). In the same way, one can obtain the right half of the tilted diamond,

$$
\begin{aligned}
\left(\Delta \phi_{E}\right)_{\text {right }}= & -\iint_{\text {right }} E_{x} \mathrm{~d} x \wedge c \mathrm{~d} t=-A_{0} \frac{\omega}{c} \int_{0}^{\Delta x} \\
& \times\left(\int_{x / v_{x}}^{2 \Delta t-x / v_{x}} f^{\prime}(\omega t \pm k z) c \mathrm{~d} t\right) \mathrm{d} x \\
= & -A_{0} \frac{\omega}{\omega^{\prime}} \int_{0}^{\Delta x}\left[f\left(2 \omega^{\prime} \Delta t-\omega^{\prime} x / v_{x}\right)\right. \\
& \left.-f\left(\omega^{\prime} x / v_{x}\right)\right] \mathrm{d} x .
\end{aligned}
$$

Next, performing the $x$ integration in (39), we find

$$
\begin{aligned}
\left(\Delta \phi_{E}\right)_{\text {right }}= & -A_{0} \frac{\omega v_{x}}{\left(\omega^{\prime}\right)^{2}}\left[F\left(2 \omega^{\prime} \Delta t\right)-F\left(\omega^{\prime} \Delta t\right)\right. \\
& \left.+F(0)-F\left(\omega^{\prime} \Delta t\right)\right]=A_{0} \frac{\beta_{x}}{k\left(1 \pm \beta_{z}\right)^{2}} \\
& \times\left[2 F\left(\omega^{\prime} \Delta t\right)-F\left(2 \omega^{\prime} \Delta t\right)-F(0)\right] \\
= & A_{0} \frac{1}{k^{\prime}\left(1 \pm \beta_{z}\right)}[2 F(\Delta \zeta)-F(2 \Delta \zeta)-F(0)] .
\end{aligned}
$$

Adding the left and right sides, we get the total electric contribution for the tilted diamond as

$\Delta \phi_{E}=\frac{2 A_{0}}{k^{\prime}\left(1 \pm \beta_{z}\right)}[2 F(\Delta \zeta)-F(0)-F(2 \Delta \zeta)]$.

From (36), this gives for the magnetic contribution

$\Delta \phi_{B}=\Delta \phi-\Delta \phi_{E}=\frac{ \pm 2 A_{0} \beta_{z}}{k^{\prime}\left(1 \pm \beta_{z}\right)}[2 F(\Delta \zeta)-F(0)-F(2 \Delta \zeta)]$.

Hence,

$\frac{\Delta \phi_{B}}{\Delta \phi_{E}}= \pm \beta_{z}$

which is the result given in (31).

\section{References}

1. Y. Aharonov, D. Bohm, Phys. Rev. 115, 484 (1959)

2. W. Ehrenberg, R.E. Siday, Proc. Phys. Soc. B 62, 8 (1949)

3. R.G. Chambers, Phys. Rev. Lett. 5, 3 (1960)

4. A. Tonomura et al., Phys. Rev. Lett. 56, 792 (1986)

5. N.G. van Kampen, Phys. Lett. A 106, 5 (1984)

6. S.M. Roy, V. Singh, Nuovo Cimento A 79, 391 (1984)

7. R.A. Brown, D. Home, Nuovo Cimento B 107, 303 (1991)

8. B. Gaveau, A.M. Nounou, L.S. Schulman, Found. Phys. 41, 1462 (2011)

9. D. Singleton, E. Vagenas, Phys. Lett. B 723, 241 (2013)

10. J. MacDougall, D. Singleton, J. Math. Phys. 55, 042101 (2014)

11. B. Lee, E. Yin, T.K. Gustafson, R. Chiao, Phys. Rev. A 45, 4319 (1992)

12. J. Hsiang, L.H. Ford, Phys. Rev. Lett. 92, 250402 (2004) 
13. L. Marton, J.A. Simpson, J.A. Suddeth, Rev. Sci. Instr. 25, 1099 (1954)

14. F.G. Werner, D. Brill, Phys. Rev. Lett. 4, 344 (1960)

15. J. Macdougall, D. Singleton, E.C. Vagenas, Phys. Lett. A 379, 1689 (2015)

16. YuV Chentsov, YuM Voronin, I.P. Demenchonok, A.N. Ageev, Opt. Zh. 8, 55 (1996)

17. A.N. Ageev, SYu. Davydov, A.G. Chirkov, Tech. Phys. Lett. 26, $392(2000)$
18. Y. Aharonov, A. Casher, Phys. Rev. Lett. 53, 319 (1984)

19. M. Bright, D. Singleton, Phys. Rev. D 91, 085010 (2015)

20. S. Coleman, Phys. Lett. 70B, 59 (1977)

21. L. Ryder, Quantum Field Theory. 2nd ed. (Cambridge University Press, Cambridge, 1996), Secs. 2.9

22. H. Flanders, Differential Forms with Applications to the Physical Sciences (Dover Publications Inc., New York, 1989)

23. A. Noguchi, Y. Shikano, K. Toyoda, S. Urabe, Nat. Commun. 5, $3868(2014)$ 